\title{
INVESTIGATION OF FLEXIBILITY CONSTANTS FOR A MULTI-SPRING MODEL: A SOLUTION FOR BUCKLING OF CRACKED MICRO/NANOBEAMS
}

\author{
Majid Akbarzadeh Khorshidi, Mahmoud Shariati \\ Ferdowsi University of Mashhad, Department of Mechanical Engineering, Mashhad, Iran \\ e-mail:mshariati44@um.ac.ir
}

\begin{abstract}
In this paper, a multi-spring model is used for modelling of the crack in a micro/nanobeam under axial compressive load based on a modified couple stress theory. This model includes an equivalent rotational spring to transmit the bending moment and an equivalent longitudinal spring to transmit the axial force through the cracked section, which leads to promotion of the modelling of discontinuities due to the presence of the crack. Moreover, this study considers coupled effects between the bending moment and axial force on the discontinuities at the cracked section. Therefore, four flexibility constants appear in the continuity conditions. In this paper, these four constants are obtained as a function of crack depth, separately. This modelling is employed to solve the buckling problem of cracked micro/nanobeams using a close-form method, Euler-Bernoulli theory and simply supported boundary conditions. Finally, the effects of flexibility constants, crack depth and crack location on the critical buckling load are studied.
\end{abstract}

Keywords: flexibility constants, multi-spring model, MCST, buckling, crack

\section{Introduction}

It is clear that presence of cracks or any other defects into any structure leads to a decrease of its capabilities. The issue of cracking in the structures is interested in both macro and small scale dimensions. Thus, presentation of an accurate and appropriate model to capture crack conditions is very important. In many studies, cracks have been modeled by means of different types of springs. The type of the spring model depends on problem type, such as the type of loading and geometry. In fact, kinds of displacements at the cracked section determine what modelling should be selected. For example, a longitudinal spring model is used when the axial displacement is dominant (Hsu et al., 2011), a rotational spring model is applicable for a wide range of problems in which the angle changes between the crack surfaces are important (Akbarzadeh Khorshidi et al., 2017; Akbarzadeh Khorshidi and Shariati, 2017b; Hasheminejad et al., 2011; Ke et al., 2009; Loya etal, 2006; Shaat et al., 2016; Torabi and Nafar Dastegerdi, 2012; Wang and Wang, 2013; Yang and Cheng, 2008). Structures under torsion incorporate a torsional spring to describe discontinuity at the cracked section (Loya et al., 2014). Rice and Levy (1972) stated that the presence of a crack leads to a local reduction in bending and extensional stiffness along the crack line. Therefore, it is more accurate to use a model which considers these two local reductions. Akbarzadeh Khorshidi and Shariati (2017a) presented buckling analysis of cracked nanobeams based on a modified couple stress theory and using a two-spring model at the cracked section. The authors used the mentioned model according to the historical background expressed by Rice and Levy (1972) and the discontinuity relations presented by Loya et al. (2009).

In majority of recent studies on static and dynamic behavior of micro/nanobeams in the presence of a micro or nano-scale crack, the flexibility constant which introduces the crack 
severity is considered as a hypothetical input. However, there are studies which formulate the severity of the crack as a function of the crack depth, the material length scale parameter and other mechanical characteristics of the beam (Shaat et al., 2016; Akbarzadeh Khorshidi et al., 2017). These papers use energy stored in the spring and compare it with the strain energy release rate at the crack surfaces.

In the present study, the two-spring model is employed to describe discontinuities at the cracked section and, consequently, four flexibility constants appear, which gives the severity of the crack. Each flexibility constant is presented as a function of crack depth (as an unknown parameter) and other parameters (given values). Therefore, the continuity relations are formulated against the crack depth. The macroscopic fracture mechanics is used for micro/nano-scale beams based on atomistic simulation models and continuum models (Joshi et al., 2010; Tsai et al., 2016; Hu et al., 2017). Then, a modified couple stress based solution is proposed for buckling analysis of the cracked beams.

\section{Modelling}

Consider an Euler-Bernoulli beam with length $L$, width $b$, thickness $h$ and a crack with depth $a$ is located at distance $L_{c}$ from the left side of the beam (Fig. 1a). In the present modelling, the cracked beam is modelled as two separate segments connected by two massless elastic longitudinal and rotational springs (Fig. 1b). Therefore, the total strain energy of the cracked beam is equal to the strain energy of these two segments plus the strain energy stored in the springs. With this explanation, the released potential energy due to the presence of the crack is equal to the strain energy stored by the springs. The continuity conditions governed between the two beam segments are defined as follows (Akbarzadeh Khorshidi and Shariati, 2017a; Loya et al., 2009)

$$
\begin{aligned}
w_{1} & =w_{2} \quad N_{1}=N_{2} & M_{1} & =M_{2} \\
\Delta \theta & =\bar{K}_{M M} M+\bar{K}_{M N} N & \Delta u & =\bar{K}_{N N} N+\bar{K}_{N M} M
\end{aligned}
$$

where $\Delta \theta$ is the difference in the rotation angles between two crack surfaces (or the angle rotated by the rotational spring) and $\Delta u$ is the longitudinal displacement occurred at the cracked section (or amount of longitudinal spring compression). $N$ and $M$ are, respectively, the axial force and the bending moment. Also, $\bar{K}_{M M}, \bar{K}_{M N}, \bar{K}_{N N}$ and $\bar{K}_{N M}$ are four coefficients to represent the coupled effects between the axial force and bending moment in discontinuity relations.
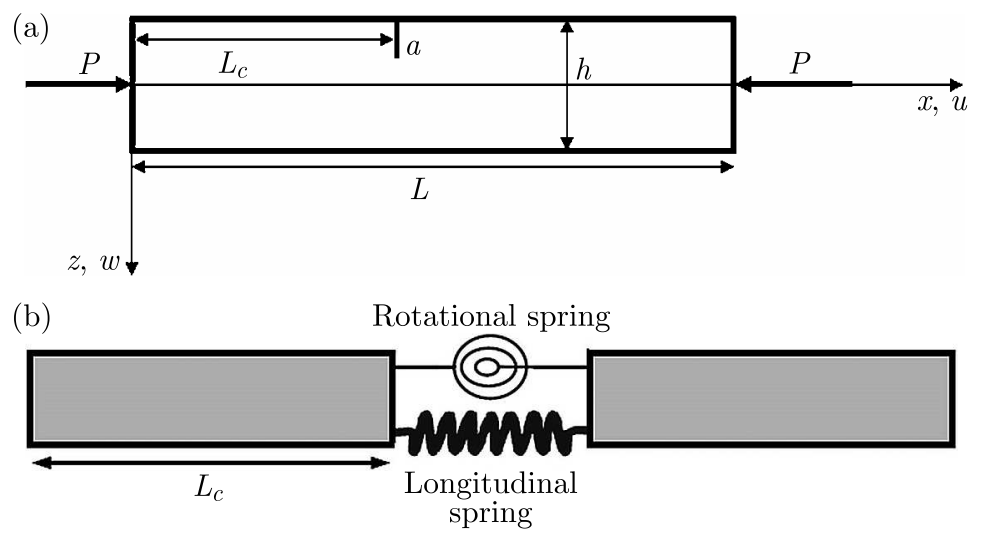

Fig. 1. (a) A schematic of the cracked beam and (b) the springs model for a cracked section

Therefore, the strain energy of springs $U_{\text {springs }}$ is stated as

$$
U_{\text {springs }}=\frac{1}{2} M \Delta \theta+\frac{1}{2} N \Delta u=\frac{1}{2} M\left(\bar{K}_{M M} M+\bar{K}_{M N} N\right)+\frac{1}{2} N\left(\bar{K}_{N M} M+\bar{K}_{N N} N\right)
$$


Based on generalized Irwin's (Irwin, 1960) relation, the potential energy-release rate $G$ is introduced as (Rice and Levy, 1972)

$$
G=\frac{\left(1-\nu^{2}\right) a}{E}\left(\pi \sigma_{b}^{2} Y_{b}^{2}+2 \sqrt{\pi} \sigma_{t} \sigma_{b} Y_{t} Y_{b}+\sigma_{t}^{2} Y_{t}^{2}\right)
$$

where $E$ is Young's modulus, $\nu$ is Poisson's ratio, $\sigma$ and $Y$, respectively, reflect the stress and a dimensionless function of the crack depth to thickness ratio $\bar{a}=a / h$. Indices $t$ and $b$ represent the status of the parameters in tension and bending, respectively.

When a cracked beam is subjected to compression, it senses a local compliance at the cracked section, and the zones around the crack tend to open the crack lips. Based on the stress concentration at the crack tip, a uniform stress field distributes along the beam thickness (see Akbarzadeh Khorshidi and Shariati, 2017b). Therefore, the crack lips suffer stretching and bending (Fig. 2). The bending stress and tensile stress (thickness average stress) defined in Eq. (2.3) are shown as

$$
\sigma_{b}=\frac{M h}{2 I}=\frac{6 M}{b h^{2}} \quad \sigma_{t}=\frac{N}{A}=\frac{N}{b h}
$$

where $I$ in Eq. $(2.4)_{1}$ represents the moment of inertia and, for a rectangular cross section, is equal to $b h^{3} / 12$. Also, $A$ in Eq. $(2.4)_{2}$ denotes the cross section area and, for the mentioned cross section, is equal to $b h$.

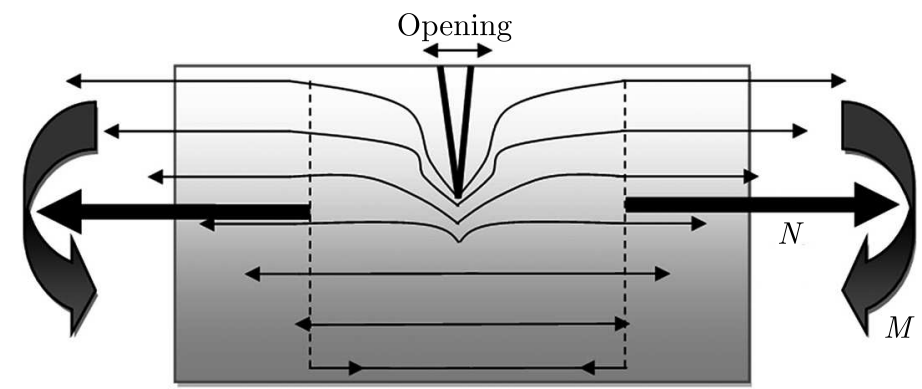

Fig. 2. The stress field due to the applied load and moment along the thickness

The strain energy due to the presence of the crack is obtained as

$$
U_{c}=\int_{0}^{a} G d A_{c}=b \int_{0}^{a} G d a
$$

Substituting Eqs. (2.3) and (2.4) into Eq. (2.5), we have

$$
U_{c}=\frac{\left(1-\nu^{2}\right)}{E b}\left(\frac{36 \pi M^{2}}{h^{2}} \int_{0}^{\bar{a}} \bar{a} Y_{b}^{2} d \bar{a}+\frac{12 \sqrt{\pi} M N}{h} \int_{0}^{\bar{a}} \bar{a} Y_{t} Y_{b} d \bar{a}+N^{2} \int_{0}^{\bar{a}} \bar{a} Y_{t}^{2} d \bar{a}\right)
$$

where $\bar{a}=a / h$ introduces the crack depth to thickness ratio. The dimensionless function $Y_{t}$ is defined as (Gross and Srawley, 1965)

$$
Y_{t}=1.99-0.41 \bar{a}+18.70 \bar{a}^{2}-38.48 \bar{a}^{3}+53.85 \bar{a}^{4}
$$

Also, the dimensionless function $Y_{b}$ is defined as (Ke et al., 2009)

$$
\begin{aligned}
Y_{b} & =1.15-1.662 \bar{a}+21.667 \bar{a}^{2}-192.451 \bar{a}^{3}+909.375 \bar{a}^{4}-2124.310 \bar{a}^{5} \\
& +2395.830 \bar{a}^{6}-1031.750 \bar{a}^{7}
\end{aligned}
$$


We consider that $U_{\text {spring }}$ represented in Eq. (2.2) is equal to $U_{c}$ obtained in Eq. (2.6), so, the flexibility constants $\bar{K}_{M M}, \bar{K}_{M N}, \bar{K}_{N N}$ and $\bar{K}_{N M}$ are separately achieved as follows

$$
\begin{array}{ll}
\bar{K}_{M M}=\frac{72 \pi\left(1-\nu^{2}\right)}{E b h^{2}} \int_{0}^{\bar{a}} \bar{a} Y_{b}^{2} d \bar{a} & \bar{K}_{M N}=\bar{K}_{N M}=\frac{12 \sqrt{\pi}\left(1-\nu^{2}\right)}{E b h} \int_{0}^{\bar{a}} \bar{a} Y_{b} Y_{t} d \bar{a} \\
\bar{K}_{N N} & =\frac{2\left(1-\nu^{2}\right)}{E b} \int_{0}^{\bar{a}} \bar{a} Y_{t}^{2} d \bar{a}
\end{array}
$$

As we know, the stress resultants introduced in Eqs. (2.1) and (2.2) (the bending moment $M$ and the axial force $N$ ) are defined as

$$
N=\int_{A} \sigma_{x x} d A \quad M=M_{1}+M_{2}=\int_{A} z \sigma_{x x} A+\int_{A} m_{x y} d A
$$

where $M_{1}$ is the conventional bending moment and $M_{2}$ is the couple moment that comes from the modified couple stress theory proposed by Yang et al. (2002). The displacement field for the Euler-Bernoulli beam is

$$
u_{1}=u(x)-z \frac{d w}{d x} \quad u_{2}=0 \quad u_{3}=w(x)
$$

where $u$ and $w$ are the axial and lateral displacements of the midplane, respectively. Therefore, the nonzero strains and stresses are shown as

$$
\varepsilon_{x x}=\frac{d u_{1}}{d x}=\frac{d u}{d x}-z \frac{d^{2} w}{d x^{2}} \quad \sigma_{x x}=E \varepsilon_{x x}=E\left(\frac{d u}{d x}-z \frac{d^{2} w}{d x^{2}}\right)
$$

Also, the nonzero terms of the symmetric curvature tensor $\chi$ and the deviatoric part of the couple stress tensor $\mathbf{m}$ are defined as (Akbarzadeh Khorshidi and Shariati, 2017a; Yang et al., 2002)

$$
\chi_{x y}=-\frac{1}{2} \frac{d^{2} w}{d x^{2}} \quad m_{x y}=-\ell^{2} \mu \frac{d^{2} w}{d x^{2}}
$$

These tensors consider the couple stress effects in the modified couple stress theory, and $\ell$ is a material length scale parameter to capture the size effect (Yang et al., 2002). $\mu=E /(2+2 \nu)$ is the shear modulus.

Now, substituting Eqs. (2.12) into Eq. (2.10), we have

$$
N=E A \frac{d u}{d x} \quad M=-\left(E I+\ell^{2} G A\right) \frac{d w^{2}}{d x^{2}}
$$

where $D_{\text {eff }}=E I+\ell^{2} G A$ is the effective beam stiffness obtained based on the modified couple stress theory. According to Eq. (2.14), we can rewrite Eq. (2.1) as

$$
\begin{aligned}
& w_{1}=w_{2} \quad \frac{d u_{1}}{d x}=\frac{d u_{2}}{d x} \quad \frac{d^{2} w_{1}}{d x^{2}}=\frac{d^{2} w_{2}}{d x^{2}} \quad \quad x=L_{c} \\
& \frac{d w_{2}}{d x}-\frac{d w_{1}}{d x}=K_{M M} \frac{d^{2} w}{d x^{2}}+K_{M N} \frac{d u}{d x} \quad u_{2}-u_{1}=K_{N N} \frac{d u}{d x}+K_{N M} \frac{d^{2} w}{d x^{2}}
\end{aligned}
$$

where

$$
\begin{array}{ll}
K_{M M}=D_{\text {eff }} \bar{K}_{M M} & K_{M N}=E A \bar{K}_{M N} \\
K_{N M}=D_{\text {eff }} \bar{K}_{N M} & K_{N N}=E A \bar{K}_{N N}
\end{array}
$$


Thus, we have

$$
\begin{aligned}
& K_{M M}=36 \pi(1-\nu) h\left[\frac{1+\nu}{6}+\left(\frac{\ell}{h}\right)^{2}\right] \int_{0}^{\bar{a}} \bar{a} Y_{b}^{2} d \bar{a} \\
& K_{M N}=12 \sqrt{\pi}\left(1-\nu^{2}\right) \int_{0}^{\bar{a}} \bar{a} Y_{b} Y_{t} d \bar{a} \\
& K_{N M}=6 \sqrt{\pi}(1-\nu) h^{2}\left[\frac{1+\nu}{6}+\left(\frac{\ell}{h}\right)^{2}\right] \int_{0}^{\bar{a}} \bar{a} Y_{b} Y_{t} d \bar{a} \\
& K_{N N}=2\left(1-\nu^{2}\right) h \int_{0}^{\bar{a}} \bar{a} Y_{t}^{2} d \bar{a}
\end{aligned}
$$

Using Eqs. (2.7) and (2.8), and integrating from Eqs. (2.16), the flexibility constants are obtained as functions of the crack depth to thickness ratio, and they are represented as follows

$$
\begin{aligned}
& K_{M M}=36 \pi(1-\nu) h\left[\frac{1+\nu}{6}+\left(\frac{\ell}{h}\right)^{2}\right] \bar{a}^{2}\left(0.6612-1.2742 \bar{a}+13.1490 \bar{a}^{2}-102.9316 \bar{a}^{3}\right. \\
& \quad+533.4547 \bar{a}^{4}-2321.1924 \bar{a}^{5}+11126.9823 \bar{a}^{6}-50267.9855 \bar{a}^{7}+175186.4492 \bar{a}^{8} \\
& \quad-4399132.5842 \bar{a}^{9}+772269.2856 \bar{a}^{10}-927343.5821 \bar{a}^{11}+723108.2196 \bar{a}^{12} \\
& \left.\quad-329586.3470 \bar{a}^{13}+66531.7539 \bar{a}^{14}\right) \\
& K_{M N}=12 \sqrt{\pi}\left(1-\nu^{2}\right) \bar{a}^{2}\left(1.1442-1.2596 \bar{a}+16.3259 \bar{a}^{2}-93.4384 \bar{a}^{3}+403.2692 \bar{a}^{4}\right. \\
& \quad-1303.1856 \bar{a}^{5}+3902.0329 \bar{a}^{6}-9790.7006 \bar{a}^{7}+17593.8331 \bar{a}^{8}-20534.4869 \bar{a}^{9} \\
& \left.\quad+14059.7654 \bar{a}^{10}-4273.8259 \bar{a}^{11}\right) \\
& K_{N M}=6 \sqrt{\pi}(1-\nu) h^{2}\left[\frac{1+\nu}{6}+\left(\frac{\ell}{h}\right)^{2}\right] \bar{a}^{2}\left(1.1442-1.2596 \bar{a}+16.3259 \bar{a}^{2}\right. \\
& \quad-93.4384 \bar{a}^{3}+403.2692 \bar{a}^{4}-1303.1856 \bar{a}^{5}+3902.0329 \bar{a}^{6}-9790.7006 \bar{a}^{7} \\
& \left.\quad+17593.8331 \bar{a}^{8}-20534.4869 \bar{a}^{9}+14059.7654 \bar{a}^{10}-4273.8259 \bar{a}^{11}\right) \\
& K_{N N}=2\left(1-\nu^{2}\right) h \bar{a}^{2}\left(1.9800-0.5439 \bar{a}+18.6485 \bar{a}^{2}-33.6968 \bar{a}^{3}+99.2611 \bar{a}^{4}\right. \\
& \left.\quad-211.9012 \bar{a}^{5}+436.8375 \bar{a}^{6}-460.4773 \bar{a}^{7}+289.9822 \bar{a}^{8}\right)
\end{aligned}
$$

\section{Solutions}

According to the Euler-Bernoulli beam theory, the governing equations for buckling of a cracked micro/nanobeam are derived as (Akbarzadeh Khorshidi and Shariati, 2017b)

$$
\begin{aligned}
& \left(E I+\ell^{2} G A\right) \frac{d^{4} w_{i}}{d x_{i}^{4}}+P \frac{d^{2} w_{i}}{d x_{i}^{2}}=0 \quad \begin{cases}i=1 & 0 \leqslant x \leqslant L_{c} \\
i=2 & L_{c} \leqslant x \leqslant L\end{cases} \\
& \frac{d^{2} u_{i}}{d x_{i}^{2}}=0 \quad \begin{cases}i=1 & 0 \leqslant x \leqslant L_{c} \\
i=2 & L_{c} \leqslant x \leqslant L\end{cases}
\end{aligned}
$$

Here the subscript $i=1,2$ refers to the left and right segments of the cracked beam. The boundary conditions of a simply supported beam are expressed as

$$
\begin{array}{lrl}
u_{1}(0)=w_{1}(0)=0 & u_{2}(L) & =w_{2}(L)=0 \\
\left.\frac{d^{2} w_{1}}{d x^{2}}\right|_{x=0}=0 & \left.\frac{d^{2} w_{2}}{d x^{2}}\right|_{x=L}=0
\end{array}
$$


The general solution to Eqs. (3.1) can be obtained as

$$
\begin{aligned}
& w_{i}(x)=A_{i} \sin (\alpha x)+B_{i} \cos (\alpha x)+C_{i} x+D_{i} \quad i=1,2 \\
& u_{i}(x)=F_{i} x+H_{i} \quad i=1,2
\end{aligned}
$$

where $\alpha=\sqrt{P / D_{\text {eff }}}, A_{i}, B_{i}, C_{i}, D_{i}, F_{i}$ and $H_{i}$ are unknown constants to be determined from the boundary and continuity conditions.

Applying continuity conditions (2.15) and boundary conditions (3.2) into Eqs. (3.3), the unknown constants are derived as

$$
\begin{aligned}
& A_{1}=\left(1-\frac{\tan (\alpha L)}{\tan \left(\alpha L_{c}\right)}\right) A_{2} \quad B_{1}=D_{1}=0 \quad C_{1}=2 \alpha \frac{\tan (\alpha L)}{\sin \left(\alpha L_{c}\right)} \frac{L-L_{c}}{L} A_{2} \\
& B_{2}=-\tan (\alpha L) A_{2} \quad C_{2}=-2 \alpha \frac{\tan (\alpha L)}{\sin \left(\alpha L_{c}\right)} \frac{L_{c}}{L} A_{2} \quad D_{2}=2 \alpha L_{c} \frac{\tan (\alpha L)}{\sin \left(\alpha L_{c}\right)} A_{2} \\
& F_{1}=F_{2} \quad H_{1}=0 \quad H_{2}=-L F_{2} \\
& F_{2}=\frac{A_{2} \alpha}{K_{M N}}\left(K_{M M} \alpha\left[\sin \left(\alpha L_{c}\right)-\tan (\alpha L) \cos \left(\alpha L_{c}\right)\right]-\frac{\tan (\alpha L)}{\sin \left(\alpha L_{c}\right)}\right)
\end{aligned}
$$

also

$$
\alpha=\frac{L+K_{N N}}{K_{M N} K_{N M}} \frac{K_{M M} \alpha\left(\sin ^{2}\left(\alpha L_{c}\right)-\frac{1}{2} \tan (\alpha L) \sin \left(2 \alpha L_{c}\right)\right)-\tan (\alpha L)}{\sin ^{2}\left(\alpha L_{c}\right)-\frac{1}{2} \tan (\alpha L) \sin \left(2 \alpha L_{c}\right)}
$$

The critical buckling load can be obtained by solving Eq. (3.5). For example, when we have an intact beam $\left(a=0 \rightarrow K_{M M}=K_{M N}=K_{N N}=K_{N M}=0\right)$, according to Eq. (3.5) we have

$$
\tan (\alpha L)=0 \rightarrow \alpha=\frac{n \pi}{L} \stackrel{n=1}{\longrightarrow} P_{c r}=D_{\text {eff }}\left(\frac{\pi}{L}\right)^{2}
$$

This is quite similar to the results obtained by Mohammad-Abadi and Daneshmehr (2014) for modified couple stress based intact microbeams.

Using Eq. (3.5), the critical buckling load corresponding to each crack depth and crack location can be determined. Also, the present model (four flexibility constants) can be compared with the common model (only one constant) by removing the other constants. Moreover, the coupled effects between the bending moment and axial force can be evaluated by neglecting the crossover flexibility constants $\left(K_{M N}\right.$ and $\left.K_{N M}\right)$.

Thus, the following equation can be used when only one flexibility constant $K_{M M}$ is employed

$$
K_{M M} L \alpha\left(\sin ^{2}\left(\alpha L_{c}\right)-\frac{1}{2} \tan (\alpha L) \sin \left(2 \alpha L_{c}\right)\right)-\tan (\alpha L)=0
$$

Also, the following equation can be used when the crossover flexibility constants are removed

$$
\left(L+K_{N N}\right) K_{M M} \alpha\left(\sin ^{2}\left(\alpha L_{c}\right)-\frac{1}{2} \tan (\alpha L) \sin \left(2 \alpha L_{c}\right)\right)-\tan (\alpha L)=0
$$

\section{Results}

To illustrate the flexibility constants effects on the buckling behavior of cracked micro/nanobeams, some numerical examples of the obtained solution are presented in this Section. Also, the effects of the crack depth and crack location on the critical buckling load are investigated. First, the obtained results are validated with (Ke et al., 2009; Wang and Quek, 
2005) for macro-scale cracked beams $(\ell=0)$. This comparison can be observed in Table 1 , so that $\bar{P}=P_{c r} / P_{c r 0}$ is the nondimensional critical buckling load (where $P_{c r 0}$ denotes the critical buckling load of an intact beam). In (Ke et al., 2009; Wang and Quek, 2005), only one flexibility constant $K_{M M}$ (one equivalent rotational spring model) was employed, so, the present results have two separate columns for the one-spring model where we have only $K_{M M}$ and the twospring model where all flexibility constants appear. In Table 1, each crack depth corresponds to the crack severity, for example, $a / h=0.1$ corresponds to $K_{M M}=0.01$ (this parameter is introduced with symbol $\Theta$ in (Ke et al., 2009)).

Table 1. Nondimensional critical buckling load $\bar{P}$ of a cracked beam $\left(L_{c}=0.5 L, \nu=0.33\right.$, $E=70 \mathrm{GPa}$ and $L=10 \mathrm{~h}$ )

\begin{tabular}{|c|c|c|c|c|}
\hline \multirow{2}{*}{$a / h$} & \multicolumn{2}{|c|}{ Present } & Ke et al. & Wang \& Quek \\
\cline { 2 - 3 } & Two springs & One spring & $(2009)$ & $(2005)$ \\
\hline \hline 0.1000 & 0.9802 & 0.9801 & 0.9809 & 0.9830 \\
\hline 0.1425 & 0.9614 & 0.9611 & 0.9622 & 0.9630 \\
\hline 0.1757 & 0.9432 & 0.9426 & 0.9442 & 0.9450 \\
\hline 0.2038 & 0.9257 & 0.9245 & 0.9266 & 0.9250 \\
\hline 0.2280 & 0.9092 & 0.9071 & 0.9096 & 0.9070 \\
\hline
\end{tabular}

Now, Table 2 and Figs. 3-6 present the critical buckling load for cracked micro/nanobeams based on the modified couple stress theory and the two-spring model. All results are obtained as a parametric study where $\nu=0.33, L / h=10$ and $\ell / h=0.5$. The present study is applicable for both micro and nano-scale problems (this issue is dependent on the scale of the material length scale parameter).

Table 2 presents nondimensional critical buckling loads for different crack depths. In this table, three types of nondimensional critical buckling loads are shown, where each load denotes a special case of the flexibility field. $\bar{P}_{1}$ is the nondimensional critical buckling load for the one-spring model where we have only $K_{M M}$ (conventional model), $\bar{P}_{2}$ is for the two-spring model, but the crossover flexibility constants are vanished (the coupled effects between the axial force and bending moment are neglected) and $\bar{P}_{3}$ is for the two-spring model where all four flexibility constants are considered. The results of Table 2 indicate that there are some differences between $\bar{P}_{3}$ and $\bar{P}_{1}$, and this discrepancy increases when the crack depth is increased. Also, comparison between $\bar{P}_{2}$ and $\bar{P}_{1}$ reveals that the use of two springs without consideration of the crossover constants has no considerable impact on the obtained results. Figure 3 approves Table 2, graphically. It is found that the two-spring model presents a greater buckling capacity of cracked beams than the conventional model. Therefore, it is found that the local flexibility at the cracked section (crack severity) caused by a particular crack depth is different for the one-spring model $\bar{P}_{1}$ and the two-spring model $\bar{P}_{3}$.

Table 2. Nondimensional critical buckling load $\bar{P}$ of cracked micro/nanobeams $\left(L_{c}=0.5 L\right)$

\begin{tabular}{|c|c|c|c|}
\hline$a / h$ & $\bar{P}_{1}$ & $\bar{P}_{2}$ & $\bar{P}_{3}$ \\
\hline \hline 0 & 1 & 1 & 1 \\
\hline 0.1 & 0.9584 & 0.9584 & 0.9586 \\
\hline 0.2 & 0.8545 & 0.8545 & 0.8567 \\
\hline 0.3 & 0.7130 & 0.7130 & 0.7232 \\
\hline 0.4 & 0.5489 & 0.5489 & 0.5772 \\
\hline 0.5 & 0.3901 & 0.3901 & 0.4504 \\
\hline
\end{tabular}




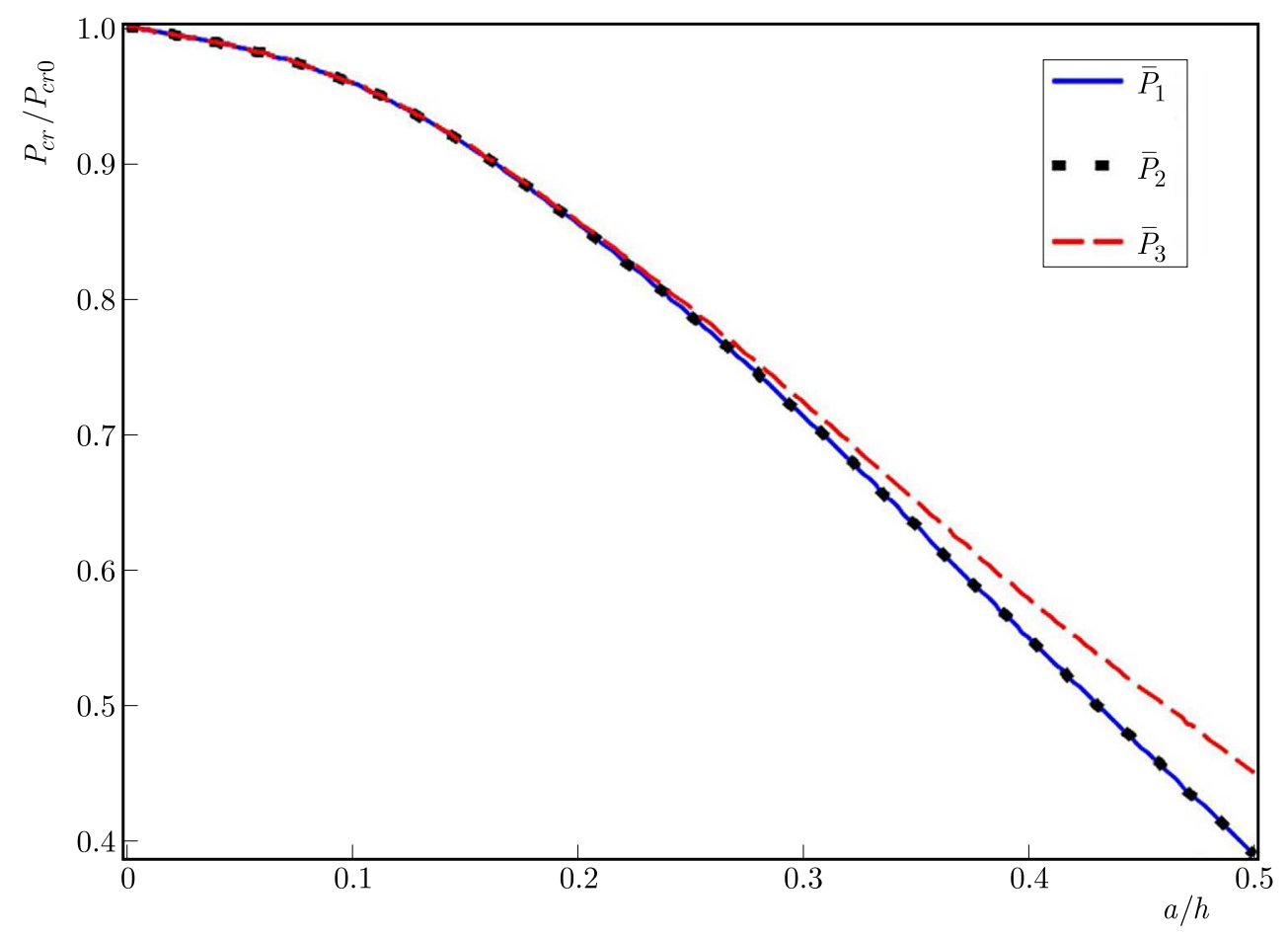

Fig. 3. Comparison of the two-spring model and the conventional model in terms of crack depth

The effect of crack location is shown in Fig. 4 for different crack depths. This figure indicates that the crack has the greatest sensitivity when it is located in middle of the beam $\left(L_{c}=0.5 L\right)$. When the crack approaches the two ends, its effect is continuously decreased. This fact is directly related to deformation of various points of the beam and, finally, the opening of the crack tip.

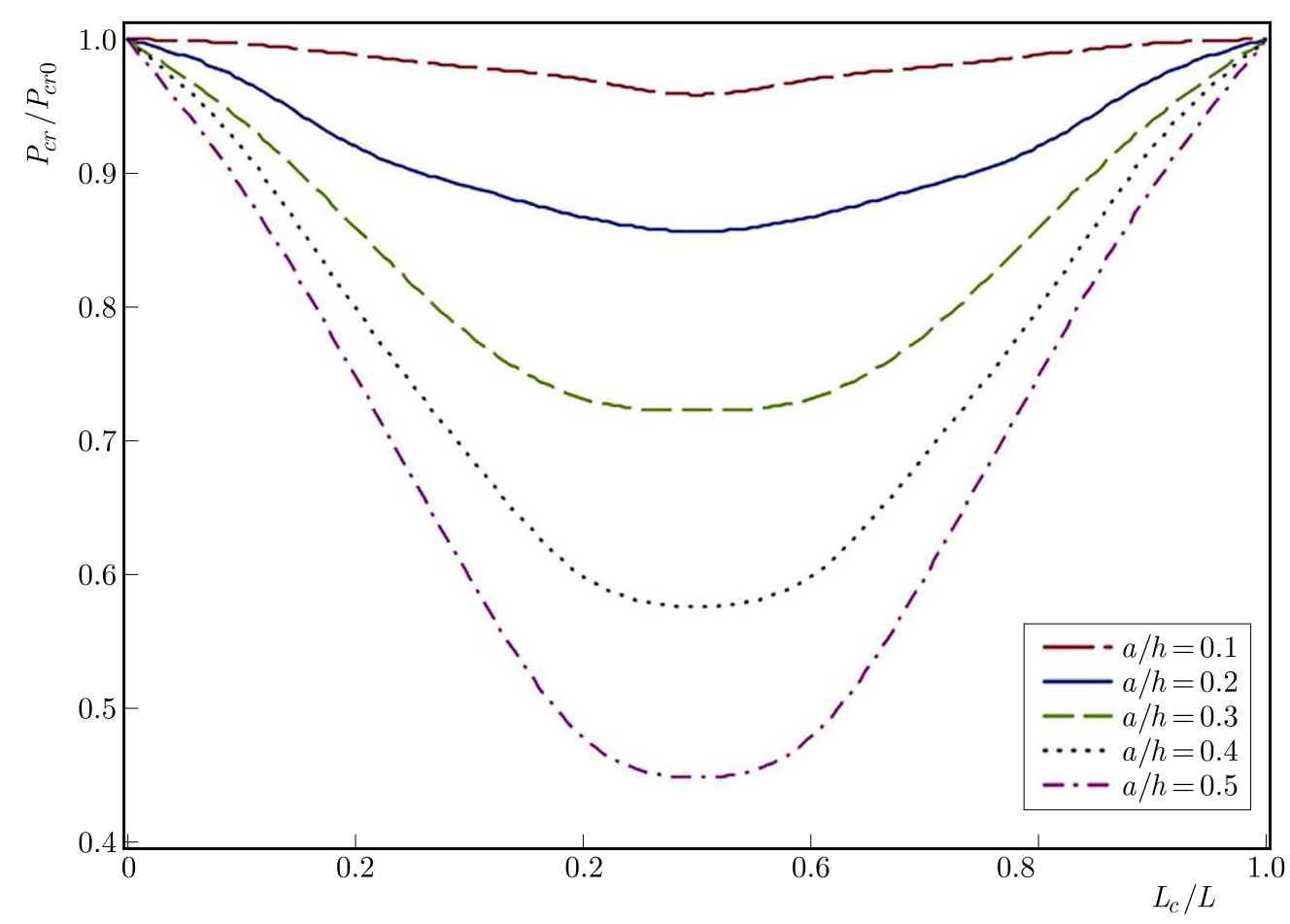

Fig. 4. The effect of crack location on the nondimensional critical buckling load with different crack depths 
Also, variations of the nondimensional critical buckling load versus crack depth are demonstrated in Fig. 5 in different crack locations. It is observed that not only the increasing of the crack depth leads to a decrease in the buckling resistance of the beam, but also makes the effect of crack location more considerable.

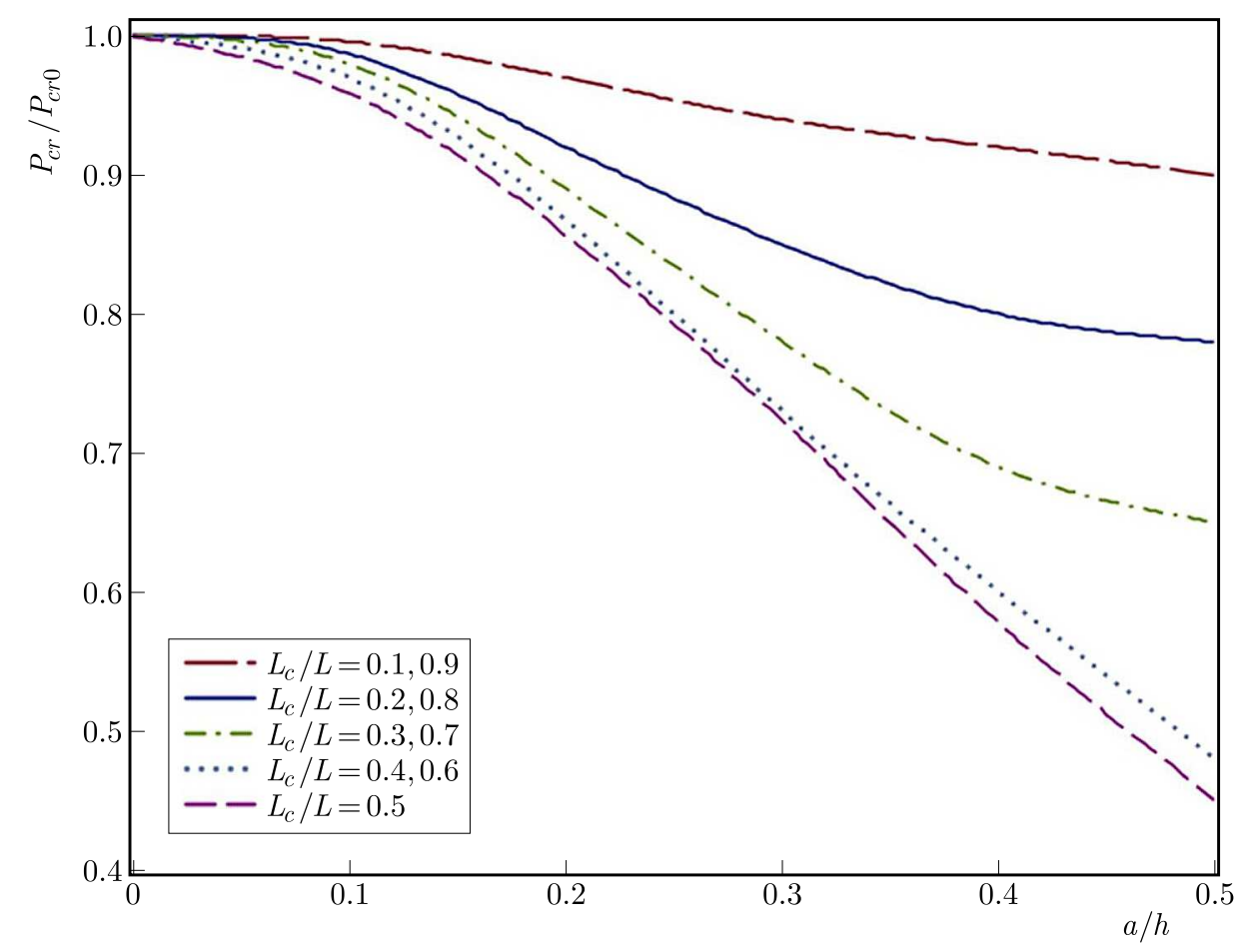

Fig. 5. The effect of crack depth on the nondimensional critical buckling load with different crack locations

\section{Conclusion}

The flexibility constants of the cracked section are investigated using a multi-spring model (rotational and longitudinal spring) to describe local flexibilities and discontinuities at the cracked section of micro/nanobeams. This model not only promotes the discontinuities but also considers the coupled effects between the bending moment and axial force on the discontinuities due to the presence of the crack. Then, the buckling problem is solved for cracked micro/nanobeams and the influence of crack depth and crack location is studied. Also, different configurations of the flexibility constants are compared together. The results show that the flexibility constant related with the bending moment (KMM) has the greatest impact on the local flexibility due to the crack (crack severity). But, this crack severity changes by adding more flexibility constants. It is found that the coupled effects between the bending moment and axial force (crossover constants) are considerable, and the making use of the multi-spring model without consideration of the crossover constants will not be useful. Therefore, the use of four constants (multi-spring model) instead of only one (conventional model) estimates the buckling capacity better, and this difference increases with an increase in the crack depth.

\section{References}

1. Akbarzadeh Khorshidi M., Shatt M., Abdelkefi A., Shariati M., 2017, Nonlocal modeling and buckling features of cracked nanobeams with von Karman nonlinearity, Applied Physics A: Material Science and Processing, 123, 62 
2. Akbarzadeh Khorshidi M., Shariati M., 2017a, A multi-spring model for buckling analysis of cracked Timoshenko nanobeams based on modified couple stress theory, Journal of Theoretical and Applied Mechanics, 55, 4, 1127-1139

3. Akbarzadeh Khorshidi M., Shariati M., 2017b, Buckling and postbuckling of size-dependent cracked microbeams based on a modified couple stress theory, Journal of Applied Mechanics and Technical Physics, 58, 4, 717-724

4. Gross B., Srawley J.E., 1965, Stress-intensity factors for single edge notch specimens in bending or combined bending and tension by boundary collocation of a stress function, NASA Technical Note, D-2603

5. Hasheminejad M., Gheshlaghi B., Mirzaei Y., Abbasion S., 2011, Free transverse vibrations of cracked nanobeams with surface effects, Thin Solid Films, 519, 2477-2482

6. Hsu J.Ch., Lee H.L., Chang W.J., 2011, Longitudinal vibration of cracked nanobeams using nonlocal elasticity theory, Current Applied Physics, 11, 1384-1388

7. Hu K.M., Zhang W.M., Peng Z.K., Meng G., 2016, Transverse vibrations of mixed-mode cracked nanobeams with surface effect, Journal of Vibration and Acoustics, 138, 1, 011020

8. Irwin G.R., 1960, Fracture mechanics, [In:] Structural Mechanics, J.N. Goodier, and N.J. Hoff (Edit.), Pergamon Press, p. 557

9. Joshi A.Y., Sharma S.C., Harsha S., 2010, Analysis of crack propagation in fixed-free singlewalled carbon nanotube under tensile loading using XFEM, ASME Journal of Nanotechnology in Engineering and Medicine, 1, 4, 041008

10. Ke L.L., Yang J., Kitipornchai S., 2009, Postbuckling analysis of edge cracked functionally graded Timoshenko beams under end shortening, Composite Structures, 90, 152-160

11. Loya J.A., Aranda-Ruiz J., Fernandez-Saez J., 2014, Torsion of cracked nanorods using a nonlocal elasticity model, Journal of Physics D: Applied Physics, 47, 115304 (12pp)

12. Loya J.A., Rubio L., Fernandez-Saez J., 2006, Natural frequencies for bending vibrations of Timoshenko cracked beams, Journal of Sound and Vibration, 290, 640-653

13. Loya J., Lopez-Puente J., Zaera R., Fernandez-Saez J., 2009, Free transverse vibrations of cracked nanobeams using a nonlocal elasticity model, Journal of Applied Physics, 105, 044309

14. Mohammad-Abadi M., Daneshmehr A.R., 2014, Size dependent buckling analysis of microbeams based on modified couple stress theory with high order theories and general boundary conditions, International Journal of Engineering Science, 74, 1-14

15. Rice J.R., Levy N., 1972, The part-through surface crack in an elastic plate, Journal of Applied Mechanics: Transaction of the ASME, March, 185-194

16. Shatt M., Akbarzadeh Khorshidi M., Abdelkefi A., Shariati M., 2016, Modeling and vibration characteristics of cracked nano-beams made of nanocrystalline materials, International Journal of Mechanical Sciences, 115-116, 574-585

17. Torabi K., Nafar Dastgerdi J., 2012, An analytical method for free vibration analysis of Timoshenko beam theory applied to cracked nanobeams using a nonlocal elasticity model, Thin Solid Films, 520, 6595-6602

18. Tsai J.L., Tzeng S.H., Tzou Y.J., 2010, Characterizing the fracture parameters of a graphene sheet using atomistic simulation and continuum mechanics, International Journal of Solids and Structures, 47, 3, 503-509

19. WANG Q., QUEK S.T., 2005, Repair of cracked column under axially compressive load via piezoelectric patch, Computers and Structures, 83, 1355-1363

20. WANG K., WANG B., 2013, Timoshenko beam model for the vibration analysis of a cracked nanobeam with surface energy, Journal of Vibration and Control, 21, 12, 2452-2464

21. Yang J., Cheng Y., 2008, Free vibration and buckling analyses of functionally graded beams with edge cracks, Composite Structures, 83, 48-60

22. Yang F., Chong A.C.M., LAm D.C.C., Tong P., 2002, Couple stress based strain gradient theory for elasticity, International Journal of Solids and Structures, 39, 2731-2743 